\title{
Stabilization with Internal Loop for Infinite-Dimensional Discrete Time-Varying Systems
}

\author{
Nai-feng Gan, ${ }^{1,2}$ Yu-feng LU, ${ }^{1}$ and Ting Gong ${ }^{1}$ \\ ${ }^{1}$ School of Mathematical Sciences, Dalian University of Technology, Dalian 116027, China \\ ${ }^{2}$ College of Mathematics and Information Science, Anshan Normal University, Anshan 114007, China \\ Correspondence should be addressed to Nai-feng Gan; smbjbm@yeah.net
}

Received 22 January 2014; Revised 9 April 2014; Accepted 10 April 2014; Published 28 April 2014

Academic Editor: Manuel De la Sen

Copyright (C) 2014 Nai-feng Gan et al. This is an open access article distributed under the Creative Commons Attribution License, which permits unrestricted use, distribution, and reproduction in any medium, provided the original work is properly cited.

\begin{abstract}
The concepts of stabilization with internal loop are analyzed for well-posed transfer functions. We obtain some sufficient and necessary conditions such that a stabilizing controller with internal loop stabilizes plant $L$. We also analyze two special subclasses of stabilizing controllers with internal loop, called canonical and dual canonical controllers, and show that all stabilizing controllers can be parameterized by a doubly coprime factorization of the original transfer function.
\end{abstract}

\section{Introduction}

Control Theory is a relevant field from the mathematical theoretical point of view as well as in many applications (see [1-6]). What is important, in particular, is the closed-loop stabilization of dynamic system under appropriate feedback control as a minimum requirement to design a well-posed feedback system. In the last twenty years, the closed-loop system whose stability is achieved by the controller with internal loop has attracted the attention of many authors (see $[7,8])$. While extending the theory of dynamic stabilization to regular linear systems (a subclass of the well-posed linear systems), it was shown in [7, Example 2.3] that even the standard observer-based controller is not a well-posed linear system and its transfer function is not well-posed. To overcome this, paper [8] proposed another definition of a stabilizing controller which is more general than that has been defined earlier, the so-called stabilizing controller with internal loop. The concept enabled a simple Youla parameterization and has some advantages which turn out to be very important for infinite-dimensional systems. It makes the theory of dynamic stabilization simpler and more natural [8].

Recently, the study of time-varying systems using modern mathematical methods has come into its own. This is a scientific necessity. After all, many common physical systems are time varying (see [9-14]). Paper [15] studied the concept of stabilization with internal loop for infinite-dimensional discrete time-varying systems and gave a parameterization of all stabilizing controllers with internal loop if $I-K_{22}$ has a wellposed inverse in the framework of nest algebra. But in many cases, the controller $C=K_{11}+K_{12}\left(I-K_{22}\right)^{-1} K_{21}$ will not be well-posed, but $C$ perhaps stabilizes $L$.

In this paper, we study the stabilization with internal loop for the linear time-varying system under the framework of nest algebra. We extend our study of controllers with internal loop to more general use and give a parameterization of all stabilizing controllers with internal loop even if $I-K_{22}=0$. It is found that the stabilization with internal loop for the linear time-varying system obtained in [15] can be viewed as a special case of that obtained here. As we know, if the plant is not strictly proper, it is difficult to choose the parameter in such way that the resulting controller will be well-posed. Even if we choose to ignore well-posedness, we still have to ensure that the denominator in the Youla parameterization is invertible. This makes it awkward to use this parameterization to solve the practical problems, while the controller with internal loop overcomes this awkwardness. We obtain canonical and dual canonical controllers and show that all stabilizing controllers can be parameterized by a doubly coprime factorization of the original transfer function.

The rest of this paper is organized as follows. Mathematical background material and notation are introduced in 
Section 2. In Section 3, we give some sufficient and necessary conditions that a stabilizing controller with internal loop stabilizes plant $L$. In Section 4, we introduce canonical and dual canonical controllers. We show that a plant $L$ is stabilizable with internal loop by a canonical (dual canonical) controller if and only if $L$ has a right coprime (left coprime) factorization. We give a complete parameterization of all (dual) canonical stabilizing controllers with internal loop. Some conclusions are drawn in Section 5.

\section{Preliminaries}

We denote by $\mathbb{Z}_{+}$the nonnegative integers and by $\mathbb{C}$ the complex numbers. Let $H$ be the complex infinite-dimensional Hilbert sequence space:

$$
l^{2}=\left\{\left(x_{0}, x_{1}, \ldots\right): x_{i} \in \mathbb{C}, \sum_{i=0}^{\infty}\left|x_{i}\right|^{2}<\infty\right\},
$$

where $|\cdot|$ denotes the standard Euclidean norm on $\mathbb{C} . H_{e}$ will denote the extended space:

$$
H_{e}=\left\{\left(x_{0}, x_{1}, x_{2}, \ldots\right): x_{i} \in \mathbb{C}\right\} .
$$

Definition 1 (see [3]). A family $N$ of closed subspaces of the Hilbert space $H$ is a complete nest if

(1) $\{0\}, H \in N$.

(2) For $N_{1}, N_{2}$, either $N_{1} \subseteq N_{2}$ or $N_{2} \subseteq N_{1}$.

(3) If $\left\{N_{\alpha}\right\}$ is a subfamily in $N$, then $\cap_{\alpha} N_{\alpha}$ and $\vee_{\alpha} N_{\alpha}$ are also in $N$.

Every subspace $N$ of $H$ is identifiable with the orthogonal projection $P_{n}$

$$
P_{n}\left(x_{0}, x_{1}, \ldots, x_{n}, x_{n+1}, \ldots\right)=\left(x_{0}, x_{1}, \ldots, x_{n}, 0, \ldots\right) .
$$

Properties (1) to (3) can be reformulated as follows.

$\left(1^{\prime}\right) 0, I \in N$.

$\left(2^{\prime}\right)$ For $P_{1}, P_{2} \in N$, either $P_{1} \leq P_{2}$ or $P_{2} \leq P_{1}$.

$\left(3^{\prime}\right)$ If $\left\{P_{\alpha}\right\}$ is a nest in $N$ which converges weakly (equivalently, strongly) to $P$, then $P \in N$.

Definition 2 (see [3]). If $N$ is a nest and $P$ is its associated family of orthogonal projections,

$$
\operatorname{Alg} P=\left\{T \in \mathfrak{E}(H),\left(I-P_{n}\right) T P_{n}=0\right\}
$$

is called a nest algebra, where $\mathfrak{E}(H)$ is the algebra of all bounded linear operators on $H$.

A linear transformation $T$ on $H_{e}$ is causal if $P_{n} T=P_{n} T P_{n}$ for $n \geq 0$.

Lemma 3 (see [3]). The following are equivalent:

(1) $\mathrm{T}$ on $H_{e}$ is stable.

(2) $T$ is causal and $T \mid H$ is a bound operator.

(3) $T$ is the extension to $H_{e}$ of an operator in $A \lg R$.
This lemma allow us to identify the algebra $S$ of stable operators on $H_{e}$ with the nest algebra $\operatorname{Alg} R$. The restriction of $T \in S$ to $H$ is in $\operatorname{Alg} R$ and the extension of $S \in \operatorname{Alg} R$ to $H_{e}$ is in $S$. $A \lg R$ and $S$ are identical.

For $L, K \in \mathfrak{E}$, the operator matrix $\left(\begin{array}{cc}I & -K \\ -L & I\end{array}\right)$ defined on $H_{e} \oplus$ $H_{e}$ is called the feedback system with plant $L$ and compensator $K$.

In Figure 1, $L$ represents a given plant (system) and $K=$ $\left(\begin{array}{ll}K_{11} & K_{12} \\ K_{21} & K_{22}\end{array}\right)$ a compensator or controller; $e_{1}, e_{2}$ denote the externally applied inputs; $u_{L}, u_{K}$ denote the inputs to the plant and compensator, respectively; and $y_{L}, y_{K}$ denote the outputs of the compensator and plant, respectively.

The closed-loop system equation are

$$
\left(\begin{array}{l}
e_{1} \\
e_{2}
\end{array}\right)=\left(\begin{array}{cc}
I & -K \\
-L & I
\end{array}\right)\left(\begin{array}{l}
u_{L} \\
u_{K}
\end{array}\right)
$$

The system is well-posed if the internal input $u$ can be expressed as a causal function of the external input $e$. This is equivalent to requiring that $\left(\begin{array}{cc}I & -K \\ -L & I\end{array}\right)$ be invertible. The inverse is easily computed formally and is given by the matrix as follows:

$$
H(L, K)=\left(\begin{array}{cc}
(I-K L)^{-1} & K(I-L K)^{-1} \\
L(I-K L)^{-1} & (I-L K)^{-1}
\end{array}\right) .
$$

The closed-loop system $\{L, K\}$ is stable if $\left(\begin{array}{cc}I & -K \\ -L & I\end{array}\right)$ has a bound causal inverse defined on $H \oplus H$. The stability of the closed-loop system is equivalent to requiring that the four elements of the $2 \times 2$ matrix $H(L, K)$ be in $S . L \in £$ is stabilizable if there exists $K \in \mathfrak{E}$ such that $\{L, K\}$ is stable.

\section{Stabilization with Internal Loop}

In this section, a new type of controller is introduced, the socalled stabilizing controller with internal loop; see [16-18].

The intuitive interpretation of Figure 2 is as follows: $L$ represents the plant and $K$ is the transfer function of the controller from $\left(\begin{array}{c}y_{k} \\ \zeta_{i}\end{array}\right)$ to $\left(\begin{array}{l}u_{k} \\ \zeta_{0}\end{array}\right)$, when all the connections are open. The connection from $\xi_{0}$ to $\xi_{i}$ is the so-called internal loop.

$$
\begin{gathered}
\text { Partitioning } K \text { into }\left(\begin{array}{ll}
K_{11} & K_{12} \\
K_{21} & K_{22}
\end{array}\right) \text { where } K_{i j} \in \mathfrak{E}, i, j=1,2, \ldots, \\
F(K, L)=\left(\begin{array}{ccc}
I & -K_{11} & -K_{12} \\
-L & I & 0 \\
0 & -K_{21} & I-K_{22}
\end{array}\right)
\end{gathered}
$$

is the transfer function of the closed-loop system from $\left(\begin{array}{c}u_{L} \\ y_{k} \\ \zeta_{i}\end{array}\right)$ to $\left(\begin{array}{c}e_{1} \\ e_{2} \\ e_{3}\end{array}\right)$.

Suppose $I-K_{22}$ is invertible in $£$; a parameterization of all stabilizing controllers with internal loop is given in [15]. If $I-K_{22}$ has a well-posed inverse, the internal loop can be closed first and the transfer function from $y_{k}$ to $u_{k}$ is

$$
C=K_{11}+K_{12}\left(I-K_{22}\right)^{-1} K_{21} \text {. }
$$

But in many cases, the expression (8) is not defined at all (this can happen if $I-K_{22}$ is nowhere invertible). 


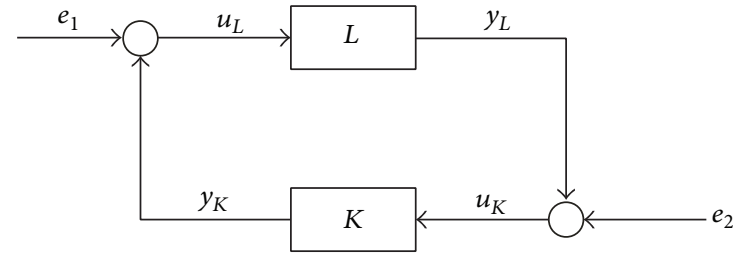

FIgURE 1: The standard feedback system.

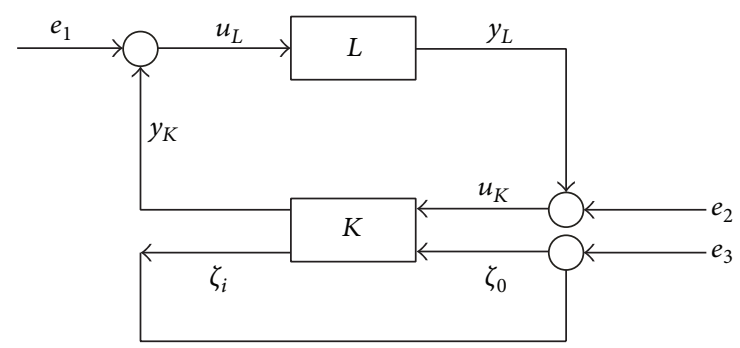

FIgURE 2: The plant $L$ connected to a controller $K$ with internal loop.

Example 4. Suppose $L=I$,

$$
\begin{aligned}
& K_{11}=0, \quad K_{12}=I, \quad K_{21}=\left(\begin{array}{ccccc}
1 & & & & \\
0 & 2 & & & \\
0 & 0 & 3 & & \\
0 & 0 & 0 & 4 & \\
\vdots & \vdots & \vdots & \vdots & \ddots
\end{array}\right) \text {, } \\
& K_{22}=\left(\begin{array}{ccccc}
1 & & & & \\
0 & 1 & & & \\
0 & 0 & 1 & & \\
0 & 0 & 0 & 1 & \\
\vdots & \vdots & \vdots & \vdots & \ddots
\end{array}\right) \text {. }
\end{aligned}
$$

It is easy to see that the transfer function (8) of the controller is undefined since $I-K_{22}=0$. It is not difficult to check that $K$ stabilizes $L$ with internal loop (this verification can be simplified considerably by using Lemma 10 ).

In the following, we give some sufficient and necessary conditions such that a stabilizing controller with internal loop stabilizes plant $L$ avoiding the condition that $I-K_{22}$ is invertible.

Theorem 5. Suppose that $K_{11}$ is an admissible feedback transfer function for $L$. Then $F(K, L)$ has a well-posed inverse if and only if $I-M$ is invertible in $€$, where $M=K_{22}+K_{21} L(I-$ $\left.K_{11} L\right)^{-1} K_{12}$.

Proof. Consider the following

$$
\begin{aligned}
F(K, L) & =\left(\begin{array}{ccc}
I & -K_{11} & -K_{12} \\
-L & I & 0 \\
0 & -K_{21} & I-K_{22}
\end{array}\right) \\
& \doteq\left(\begin{array}{ll}
S_{11} & S_{12} \\
S_{21} & S_{22}
\end{array}\right)=\left(\begin{array}{ll}
S_{11} & 0 \\
S_{21} & I
\end{array}\right)\left(\begin{array}{cc}
I & S_{11}^{-1} S_{12} \\
0 & \Delta
\end{array}\right),
\end{aligned}
$$

where $S_{11}=\left(\begin{array}{cc}I & -K_{11} \\ -L & I\end{array}\right), S_{12}=\left(\begin{array}{c}-K_{12} \\ 0\end{array}\right), S_{21}=\left(\begin{array}{ll}0 & -K_{21}\end{array}\right), S_{22}=$ $I-K_{22}, \Delta=S_{22}-S_{21} S_{11}^{-1} S_{12}$.

Since $S_{11}=\left(\begin{array}{cc}I & -K_{11} \\ -L & I\end{array}\right)$ is invertible in $M_{2}(\mathfrak{E})$, thus $\left(\begin{array}{ccc}I & -K_{11} & -K_{12} \\ -L & I \\ 0 & -K_{21} & I-K_{22}\end{array}\right)$ is invertible in $M_{3}(\mathfrak{E})$ if and only if

$$
\begin{aligned}
\Delta= & S_{22}-S_{21} S_{11}^{-1} S_{12}=I-K_{22}-\left(\begin{array}{ll}
0 & -K_{21}
\end{array}\right) \\
& \times\left(\begin{array}{cc}
I & -K_{11} \\
-L & I
\end{array}\right)^{-1}\left(\begin{array}{c}
-K_{12} \\
0
\end{array}\right)=I-M
\end{aligned}
$$

is invertible in $\mathfrak{E}$.

Further, the condition that $F(K, L)$ has a well-posed inverse is equivalent to that $K$ is an admissible feedback transfer function with internal loop for $L$ [7], so we have the following result.

Theorem 6. Suppose that $K_{11}$ is a stabilizing controller for $L$; then $K=\left(\begin{array}{ll}K_{11} & K_{12} \\ K_{21} & K_{22}\end{array}\right)$ is a stabilizing controller with internal loop for $L$ if and only if

(i) $(I-M)^{-1} \in S$, where $M=K_{22}+K_{21} L\left(I-K_{11} L\right)^{-1} K_{12}$,

(ii) there exist $E_{1}, E_{2} \in S$ such that $L E_{1} \in S, E_{2} L \in S$, $E_{1}(I-M) E_{2} L \in S, E_{1}(I-M) E_{2} \in S, L E_{1}(I-M) E_{2} \in S$, $L E_{1}(I-M) E_{2} L \in S$,

(iii) $K_{12}=\left(I-K_{11} L\right) E_{1}(I-M)$,

(iv) $K_{21}=(I-M) E_{2}\left(I-L K_{11}\right)$.

Proof. $K_{11}$ stabilizes $L$ if and only if $\left(\begin{array}{cc}\left(I-K_{11} L\right)^{-1} & K_{11}\left(I-L K_{11}\right)^{-1} \\ L\left(I-K_{11} L\right)^{-1} & \left(I-L K_{11}\right)^{-1}\end{array}\right) \epsilon$ $M_{2}(S)$.

If there exist $E_{1}, E_{2} \in S$ that satisfy (i)-(iv), all components in $H(L, K)=F(K, L)^{-1}$ are

$$
\begin{aligned}
(1,1)= & \left(I-K_{11} L\right)^{-1} K_{12}(I-M)^{-1} K_{21} L\left(I-K_{11} L\right)^{-1} \\
& +\left(I-K_{11} L\right)^{-1} \\
= & \left(I-K_{11} L\right)^{-1}\left(I-K_{11} L\right) \\
& \times E_{1}(I-M)(I-M)^{-1}(I-M) E_{2}\left(I-L K_{11}\right) \\
& \times L\left(I-K_{11} L\right)^{-1}+\left(I-K_{11} L\right)^{-1} \\
= & \left(I-K_{11} L\right)^{-1}\left(I-K_{11} L\right) E_{1}(I-M) \\
& \times(I-M)^{-1}(I-M) E_{2}\left(L-L K_{11} L\right) \\
& \times\left(I-K_{11} L\right)^{-1}+\left(I-K_{11} L\right)^{-1} \\
= & \left(I-K_{11} L\right)^{-1}\left(I-K_{11} L\right) E_{1}(I-M) \\
& \times(I-M)^{-1}(I-M) E_{2} L\left(I-K_{11} L\right) \\
& \times\left(I-K_{11} L\right)^{-1}+\left(I-K_{11} L\right)^{-1} \\
= & E_{1}(I-M) E_{2} L+\left(I-K_{11} L\right)^{-1} \in S,
\end{aligned}
$$




$$
\begin{aligned}
& (1,2)=\left(I-K_{11} L\right)^{-1} K_{11}+E_{1}(I-M) E_{2} \in S, \\
& (1,3)=E_{1} \in S \\
& (2,1)=L E_{1}(I-M) E_{2} L+L\left(I-K_{11} L\right)^{-1} \in S, \\
& (2,2)=\left(I-L K_{11}\right)^{-1}+L E_{1}(I-M) E_{2} \in S, \\
& (2,3)=L E_{1} \in S \\
& (3,1)=E_{2} L \in S \\
& (3,2)=E_{2} \in S \\
& (3,3)=(I-M)^{-1} \in S .
\end{aligned}
$$

Thus, $H(L, K) \in M_{3}(S),\{L, K\}$ is stable.

Conversely, $H(L, K)=F(K, L)^{-1}$, and all components are

$$
\begin{aligned}
& (1,1)=\left(I-K_{11} L\right)^{-1}\left[K_{12}(I-M)^{-1} K_{21} L\left(I-K_{11} L\right)^{-1}+I\right], \\
& (1,2)=\left(I-K_{11} L\right)^{-1}\left[K_{11}+K_{12}(I-M)^{-1} K_{21}\left(I-L K_{11}\right)^{-1}\right], \\
& (1,3)=\left(I-K_{11} L\right)^{-1} K_{12}(I-M)^{-1}, \\
& (2,1)=L\left(I-K_{11} L\right)^{-1}\left[K_{12}(I-M)^{-1} K_{21} L\left(I-K_{11} L\right)^{-1}+I\right], \\
& (2,2)=\left(I-L K_{11}\right)^{-1}\left[I+L K_{12}(I-M)^{-1} K_{21}\left(I-L K_{11}\right)^{-1}\right], \\
& (2,3)=L\left(I-K_{11} L\right)^{-1} K_{12}(I-M)^{-1}, \\
& (3,1)=(I-M)^{-1} K_{21} L\left(I-K_{11} L\right)^{-1}, \\
& (3,2)=(I-M)^{-1} K_{21}\left(I-L K_{11}\right)^{-1}, \\
& (3,3)=(I-M)^{-1},
\end{aligned}
$$

where $M=K_{22}+K_{21} L\left(I-K_{11} L\right)^{-1} K_{12}$. If $H(L, K) \in M_{3}(S)$, then $(I-M)^{-1} \in S$. Let $\left(I-K_{11} L\right)^{-1} K_{12}(I-M)^{-1}=E_{1} \in S$, $(I-$ $M)^{-1} K_{21}\left(I-L K_{11}\right)^{-1}=E_{2} \in S$; then $K_{12}=\left(I-K_{11} L\right) E_{1}(I-$ $M), K_{21}=(I-M) E_{2}\left(I-L K_{11}\right)$. From $(3,1) \in S$ and $(2,3) \in S$, we have $E_{2} L \in S$ and $L E_{1} \in S$. Consider $(1,1) \in S,(1,2) \in S$, $(2,1) \in S,(2,2) \in S$, and $\left\{L, K_{11}\right\}$ are stable; thus all other conditions in (ii) hold.

Remark 7. $\left\{L, K_{11}\right\}$ stable is only sufficient condition for $\{L, K\}$ stable, but not a necessary condition.

Theorem 8. If $K_{11}$ is an admissible controller for $P$, then $\{L, K\}$ is stable if and only if

(i) $\Delta^{-1}=\left[I-K_{22}-K_{21} L\left(I-K_{11} L\right)^{-1} K_{12}\right]^{-1} \in S$,

(ii) $A=\left(I-K_{11} L\right)^{-1} K_{12} \Delta^{-1} K_{21}\left(I-L K_{11}\right)^{-1}+K_{11}(I-$ $\left.L K_{11}\right)^{-1}=\left(I-K_{11} L\right)^{-1}\left(K_{12} \Delta^{-1} K_{21}+K_{11}-\right.$ $\left.K_{11} L K_{11}\right)\left(I-L K_{11}\right)^{-1} \in S$,

(iii) $A L \in S, L A \in S, L A L+L \in S, K_{21}\left(I-L K_{11}\right)^{-1} L \in S$, $L\left(I-K_{11} L\right)^{-1} K_{12} \in S, K_{21}\left(I-L K_{11}\right)^{-1} \in S,(I-$ $\left.K_{11} L\right)^{-1} K_{12} \in S$.
In fact, the conditions of Theorem 8 are weaker than those of Theorem 6. From the proof of Theorem 6, it is easy to obtain the result of Theorem 8 .

We extend the plant $G=\left(\begin{array}{ccc}\dot{L} & 0 & 0 \\ 0 & K_{11} & K_{12} \\ 0 & K_{21} & K_{22}\end{array}\right)$, and $L$ and $C$ are parallel connection. $F=\left(\begin{array}{lll}0 & I & 0 \\ I & 0 & 0 \\ 0 & 0 & I\end{array}\right)$ as a feedback operator of $G$, so we have the following result.

Theorem 9. $K$ is a stabilizing controller with internal loop for $L$ if and only if I - FG is invertible in $M_{3}(S)$.

Proof. $F$ is a stabilizing controller for $G$ if and only if

$$
\left(\begin{array}{cc}
(I-F G)^{-1} & F(I-G F)^{-1} \\
G(I-F G)^{-1} & (I-G F)^{-1}
\end{array}\right) \in M_{6}(S) .
$$

If $(I-F G)^{-1} \in M_{3}(S)$, then $F(I-G F)^{-1}=(I-F G)^{-1} F \in$ $M_{3}(S)$. Since $(I-G F)^{-1}=I+G(I-F G)^{-1} F$, thus we only need to prove $G(I-F G)^{-1} \in M_{3}(S)$. Consider $F^{2}=I$; thus $G(I-$ $F G)^{-1}=F^{2} G(I-F G)^{-1}=F\left[F G(I-F G)^{-1}\right]=F\left[(I-F G)^{-1}-\right.$ $\left.(I-F G)(I-F G)^{-1}\right]=F(I-F G)^{-1}-F$. If $(I-F G)^{-1} \in M_{3}(S)$, then $G(I-F G)^{-1} \in M_{3}(S)$.

Conversely, it is obvious.

\section{Canonical and Dual Canonical Controllers}

Another motivation for introducing controllers with internal loop is to obtain Youla parameterization. If the plant is not strictly proper, it is difficult to choose the parameter in such way that the resulting controller will be well-posed. Even if we choose to ignore well-posedness, we still have to ensure that the denominator in the Youla parameterization is invertible. By contrast, we can obtain a parameterization for all stabilizing canonical or dual canonical controllers.

The transfer functions of the controllers obtained there were of the form

$$
K=\left(\begin{array}{cc}
0 & I \\
K_{21} & K_{22}
\end{array}\right), \quad \text { with } K_{21}, K_{22} \in S .
$$

We call the controllers of form (15) canonical controllers. Analogously, controllers of the form

$$
K=\left(\begin{array}{ll}
0 & K_{12} \\
I & K_{22}
\end{array}\right), \quad \text { with } K_{12}, K_{22} \in S
$$

will be called dual canonical controllers.

In following, we analyze the properties of (dual) canonical controllers in some detail. First, we recall Lemma 10 from [15].

Lemma 10 (see [15]). The canonical controller $K=\left(\begin{array}{cc}0 & I \\ K_{21} & K_{22}\end{array}\right)$ stabilizes $L \in £$ with internal loop if and only if

$$
\Delta=I-K_{22}-K_{21} L
$$

is invertible in $S$ and $L \Delta^{-1} \in S$.

If $L \in £$ has a right-coprime factorization $L=N M^{-1}$, then $K$ stabilizes $L$ with internal loop if and only if

$$
D=M-K_{22} M-K_{21}
$$

is invertible in $S$. 
We now turn to the problem of simultaneous stabilization. Given $L_{0} \in S$ and $L_{1} \in \mathfrak{E}$, the following Corollaries 11 and 12 give the conditions that $L_{1}-L_{0}$ can be stabilized by some canonical controller.

Corollary 11. If $L_{0} \in S$ and $L_{1} \in £$ can be simultaneously stabilized by canonical controller $K=\left(\begin{array}{cc}0 & I \\ K_{21} & K_{22}\end{array}\right)$, then $L_{1}-L_{0}$ can be strongly stabilized by some canonical controller.

Proof. If $\left(\begin{array}{c}M_{1} \\ N_{1}\end{array}\right)$ is a strong right representation of $L_{1}$, then $\left(\begin{array}{c}M_{1} \\ N_{1}-L_{0} M_{1}\end{array}\right)$ is a strong right representation of $L_{1}-L_{0}$, since

$$
\begin{aligned}
& \left(Y_{1}+X_{1} L_{0}\right) M_{1}+X_{1}\left(N_{1}-L_{0} M_{1}\right) \\
& \quad=Y_{1} M_{1}+X_{1} L_{0} M_{1}+X_{1} N_{1}-X_{1} L_{0} M_{1}=I,
\end{aligned}
$$

for $L_{0} \in S$.

Suppose $R=\left(\begin{array}{cc}0 & I \\ R_{21} & R_{22}\end{array}\right)$ stabilizes $L_{1}-L_{0}$; then by Lemma 10,

$$
\begin{aligned}
D^{\prime} & =M_{1}-R_{22} M_{1}+R_{21}\left(N_{1}-L_{0} M_{1}\right) \\
& =\left(I-R_{22}-R_{21} L_{0}\right) M_{1}+R_{21} N_{1}
\end{aligned}
$$

is invertible in $S$. By Lemma $10, \Delta$ and $D$ are invertible in $S$ :

$$
\begin{aligned}
\Delta D= & \left(I-K_{22}-K_{21} L_{0}\right)\left(M_{1}-K_{22} M_{1}-K_{21} N_{1}\right) \\
= & \left(I-2 K_{22}-K_{21} L_{0}+K_{22}^{2}+K_{21} L_{0} K_{22}\right) M_{1} \\
& +\left(-I+K_{22}+K_{21} L_{0}\right) K_{21} N_{1} .
\end{aligned}
$$

Define

$$
\begin{aligned}
R_{21}= & \left(-I+K_{22}+K_{21} L_{0}\right) K_{21} \in S, \\
R_{22}= & 2 K_{22}+2 K_{21} L_{0}-K_{22}^{2}-K_{21} L_{0} K_{22} \\
& -K_{22} K_{21} L_{0}-K_{21} L_{0} K_{21} L_{0} \in S .
\end{aligned}
$$

Thus $D^{\prime}$ is invertible in $S$, and $R=\left(\begin{array}{cc}0 & I \\ R_{21} & R_{22}\end{array}\right)$ stabilizes $L_{1}-$ $L_{0}$.

Corollary 12. Suppose $L_{0} \in S, L_{1} \in £$, and $\left(\begin{array}{c}M_{1} \\ N_{1}\end{array}\right)$ is a strong right representation of $L_{1}$. If $L_{1}$ can be stabilized by canonical controller $K=\left(\begin{array}{cc}0 & I \\ K_{21} & K_{22}\end{array}\right)$, then $L_{1}-L_{0}$ can be stabilized by some canonical controller.

Proof. Since $L_{0} \in S$, then $\left(\begin{array}{c}M_{1} \\ N_{1}-L_{0} M_{1}\end{array}\right)$ is a strong right representation of $L_{1}-L_{0}$. By Lemma $10, K=\left(\begin{array}{cc}0 & I \\ K_{21} & K_{22}\end{array}\right)$ stabilizes $L_{1}$ if and only if $D=M_{1}-K_{22} M_{1}-K_{21} N_{1}$ is invertible in $S$. Suppose $R=\left(\begin{array}{cc}0 & I \\ R_{21} & R_{22}\end{array}\right)$ stabilizes $L_{1}-L_{0}$; then by Lemma 10 ,

$$
\begin{aligned}
D^{\prime} & =M_{1}-R_{22} M_{1}-R_{21}\left(N_{1}-L_{0} M_{1}\right) \\
& =\left(I-R_{22}+R_{21} L_{0}\right) M_{1}-R_{21} N_{1}
\end{aligned}
$$

is invertible in $S$. Define $R_{21}=K_{21} \in S, R_{22}=K_{22}+K_{21} L_{0} \in S$; thus $D^{\prime}$ is invertible in $S$, and $R=\left(\begin{array}{cc}0 & I \\ R_{21} & R_{22}\end{array}\right)$ stabilizes $L_{1}-$ $L_{0}$.
The conditions of Corollary 12 are weaker than those of Corollary 11. In following, we will discuss the stabilization of $\{L, K\}$ with coprime factorizations.

Theorem 13. The canonical controller $\left(\begin{array}{cc}0 & I \\ K_{21} & K_{22}\end{array}\right)$ stabilizes $L$ if and only if $\Delta=I-K_{22}-K_{21} L \in £$ is invertible in $S$ and $L \Delta^{-1} \in S$.

Proof. Let $K_{11}=0, K_{12}=I, K_{21}, K_{22} \in S$; from Theorem 8, we have that $\Delta=I-K_{22}-K_{21} L \in £$ is invertible in $S$ and $L \Delta^{-1} \in S$.

Remark 14. When $K_{11}=0,\left(\begin{array}{cc}\left(I-K_{11} L\right)^{-1} & K_{11}\left(I-L K_{11}\right)^{-1} \\ L\left(I-K_{11} L\right)^{-1} & \left(I-L K_{11}\right)^{-1}\end{array}\right)=$ $\left(\begin{array}{ll}I & 0 \\ L & I\end{array}\right) \in M_{2}(\mathfrak{E})$, thus $K_{11}=0$ is an admissible controller for $L$; we do not need to emphasize this in Theorem 13.

Remark 15. By Remark 14, $L \in £$, but $L \bar{\epsilon} S, K_{11}=0$ is not a stabilizing controller for $L$, but $\left(\begin{array}{cc}0 & I \\ K_{21} & K_{22}\end{array}\right)$ is a stabilizing controller with internal loop for $L$.

Theorem 16. If L has right coprime factorization $N M^{-1}$, then $L$ can be stabilized by canonical controller $\left(\begin{array}{cc}0 & I \\ K_{21} & K_{22}\end{array}\right)$ if and only if $M-K_{22} M-K_{21} N$ is invertible in $S$.

Proof. By Theorem 13, $\{L, K\}$ is stable if and only if $\Delta^{-1}$, $L \Delta^{-1} \in S$. Consider $L=N M^{-1}$; then $\Delta^{-1}=M\left(M-K_{22} M-\right.$ $\left.K_{21} N\right)^{-1}, L \Delta^{-1}=N\left(M-K_{22} M-K_{21} N\right)^{-1}$. If $M-K_{22} M-$ $K_{21} N \in S$, then $\Delta^{-1}, L \Delta^{-1} \in S$. Conversely, if $\Delta^{-1}, L \Delta^{-1} \in S$ and $\left(\begin{array}{ll}Y X\end{array}\right)\left(\begin{array}{c}\Delta^{-1} \\ L \Delta^{-1}\end{array}\right)=I$, then $\left(\begin{array}{l}Y X\end{array}\right)\left(\begin{array}{c}\Delta^{-1} \\ L \Delta^{-1}\end{array}\right)=\left(M-K_{22} M-\right.$ $\left.K_{21} N\right)^{-1} \in S$.

Theorem 17. If L has right coprime factorization $N M^{-1}$ if and only if L can be stabilized by some canonical controller.

Proof. If $N M^{-1}$ is right coprime factorization of $L$, there exist $Y, X \in S$ such that $\left(\begin{array}{ll}Y & X\end{array}\right)\left(\begin{array}{l}M \\ N\end{array}\right)=I$. Take $K_{21}=-X \in S, K_{22}=$ $I-Y \in S$; then $M-K_{22} M-K_{21} N=Y M+X N=I$ is invertible in $S$. By Theorem 13, ( $\left.\begin{array}{cc}0 & I \\ -X & I-Y\end{array}\right)$ stabilizes $L$.

Conversely, If $K=\left(\begin{array}{cc}0 & I \\ K_{21} & K_{22}\end{array}\right)$ stabilizes $L$, by Theorem 13, $\Delta^{-1} \in S, L \Delta^{-1} \in S$. Take $M=\Delta^{-1}, N=L \Delta^{-1}, Y=I-K_{22} \in S$, $X=-K_{21} \in S$; then $Y M+X N=\left(I-K_{22}\right) \Delta^{-1}-K_{21} L \Delta^{-1}=I$; thus, $N M^{-1}$ is right coprime factorization of $L$.

We expect a strong relationship between stabilization with internal loop and the usual concept of stabilization by the parameterization of all stabilizing (dual) canonical controllers.

Theorem 18. Suppose that $L$ has a doubly coprime factorization; then all canonical controllers that stabilize L with internal loop are parameterized by

$$
K=\left(\begin{array}{cc}
0 & I \\
E(-X+Q \widehat{M}) & I-E(Y+Q \widehat{N})
\end{array}\right)
$$

where $Q \in S, E \in S \cap S^{-1}$. 
Proof. Take $K_{21}=E(-X+Q \widehat{M}), K_{22}=I-E(Y+Q \widehat{N})$, where $E \in S \cap S^{-1}, Q \in S$; then $D=M-K_{22} M-K_{21} N=M-$ $(I-E(Y+Q \widehat{N})) M-E(-X+Q \widehat{M}) N=E \in S \cap S^{-1}$; by Theorem $17, K$ stabilizes $L$.

Conversely, if $K$ stabilizes $L$, by Theorem 16, $D=M-$ $K_{22} M-K_{21} N$ is invertible in $S$. Consider $I=D^{-1}\left(I-K_{22}\right) M-$ $D^{-1} K_{21} N$; thus $\left(D^{-1}\left(I-K_{22}\right)-D^{-1} K_{21}\right) \in M_{1 \times 2}(S)$ is a left inverse of $\left(\begin{array}{c}M \\ N\end{array}\right)$. By Theorem 17, there exist $Q \in S$ such that $D^{-1}\left(I-K_{22}\right)=Y+Q \widehat{N},-D^{-1} K_{21}=X-Q \widehat{M}$, rewrite these as $K_{21}=D(-X+Q \widehat{M}), K_{22}=I-D(Y+Q \widehat{N})$.

The following Theorem contains the dual statements of Theorems 13, 16, 17 and 18 .

Theorem 19. (a) The dual canonical controller $\left(\begin{array}{cc}0 & \widehat{K}_{12} \\ I & \widehat{K}_{22}\end{array}\right)$ stabilizes $L$ if and only if $\widehat{\Delta}=I-\widehat{K}_{22}-L \widehat{K}_{21} \in £$ is invertible in $S$ and $\widehat{\Delta}^{-1} L \in S$.

(b) If L has left coprime factorization $\widehat{M}^{-1} \widehat{N}$, then $L$ can be

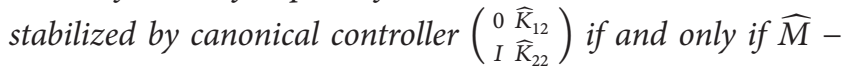
$\widehat{M} \widehat{K}_{22}-\widehat{N} \widehat{K}_{21}$ is invertible in $S$.

(c) If L has left coprime factorization $\widehat{M}^{-1} \widehat{N}$ if and only if $L$ can be stabilized by some dual canonical controller.

(d) Suppose that L has a doubly coprime factorization, then all dual canonical controllers that stabilize L with internal loop are parameterized by

$$
K=\left(\begin{array}{cc}
0 & (-\widehat{X}+M Q) E \\
I & I-(\widehat{Y}+N Q) E
\end{array}\right)
$$

where $Q \in S, E \in S \cap S^{-1}$.

The Proof of (c). Suppose $L=\widehat{M}^{-1} \widehat{N}$, there exist $\widehat{X}, \widehat{Y} \in S$ such that $(-\widehat{N} \widehat{M})\left(\begin{array}{c}-\widehat{X} \\ \widehat{Y}\end{array}\right)=I$. Let $K_{12}=-\widehat{X}, \widehat{K}_{22}=I-\widehat{Y} \in S$, then $\widehat{M}-\widehat{M} \widehat{K}_{22}-\widehat{N} \widehat{K}_{12}=I \in S \cap S^{-1}, L$ can be stabilized by $\left(\begin{array}{cc}0 & -\widehat{X} \\ I & I-\widehat{Y}\end{array}\right)$.

Conversely, if $L$ can be stabilized by $\widehat{K}$, by $(1), \widehat{\Delta}^{-1}, \widehat{\Delta}^{-1} L \epsilon$ $S$. Let $\widehat{M}=\widehat{\Delta}^{-1}, \widehat{N}=\widehat{\Delta}^{-1} L \in S, \widehat{X}=-\widehat{K}_{12}, \widehat{Y}=I-\widehat{K}_{22}$, then $L=\widehat{M}^{-1} \widehat{N}=\left(\widehat{\Delta}^{-1}\right)^{-1} \widehat{\Delta}^{-1} L, \widehat{N} \widehat{X}+\widehat{M} \widehat{Y}=I$, thus $\left(-\widehat{\Delta}^{-1} L \widehat{\Delta}^{-1}\right)$ is a left coprime factorization of $L$.

Theorem 20. If the canonical controller $\left(\begin{array}{cc}0 & I \\ K_{21} & K_{22}\end{array}\right)$ stabilizes $L$, then $L$ can be stabilized by the dual canonical controller $\left(\begin{array}{cc}0 & \Delta^{-1} K_{21} \widehat{M}^{-1} \\ I & I-\left(I+L \Delta^{-1} K_{21}\right) \widehat{M}^{-1}\end{array}\right)$.

Proof. If $L$ can be stabilized by the canonical controller $\left(\begin{array}{cc}0 & I \\ K_{21} & K_{22}\end{array}\right)$, by Theorems 13 and $17, \Delta^{-1}=\left(I-K_{22}-K_{21} L\right)^{-1} \in S$, $L \Delta^{-1} \in S$, and $L$ has a right coprime factorization. From [17], we known that $L$ has a left coprime factorization $\widehat{M}^{-1} \widehat{N}$ and there exist $\widehat{X}, \widehat{Y} \in S$ such that $\widehat{N} \widehat{X}+\widehat{M} \widehat{Y}=I$. Let $\widehat{K}_{12}=$ $\Delta^{-1} K_{21} \widehat{M}^{-1}, \widehat{K}_{22}=I-\left(I+L \Delta^{-1} K_{21}\right) \widehat{M}^{-1}$. In the following, we need to prove (1) $\widehat{K}_{12}, \widehat{K}_{22} \in S$ and (2) $\widehat{K}$ stabilizes $L$.

$\widehat{K}_{12}=\Delta^{-1} K_{21} \widehat{M}^{-1} I=\Delta^{-1} K_{21} \widehat{M}^{-1}(\widehat{N} \widehat{X}+\widehat{M} \widehat{Y})=$ $\Delta^{-1} K_{21} \widehat{M}^{-1} \widehat{N} \widehat{X}+\Delta^{-1} K_{21} \widehat{Y}=\Delta^{-1} K_{21} L \widehat{X}+\Delta^{-1} K_{21} \widehat{Y}=$ $\left[\Delta^{-1}\left(I-K_{22}\right)-I\right] \widehat{X}+\Delta^{-1} K_{21} \widehat{Y} \in S$.
Since $\left(I+L \Delta^{-1} K_{21}\right) \widehat{M}^{-1}=\left(I+L \Delta^{-1} K_{21}\right) \widehat{M}^{-1}(\widehat{N} \widehat{X}+$ $\widehat{M} \widehat{Y})=\left(L+L \Delta^{-1} K_{21} L\right) \widehat{X}+\left(I+L \Delta^{-1} K_{21}\right) \widehat{Y}=L \Delta^{-1}\left(I-K_{22}\right) \widehat{X}+$ $\left(I+L \Delta^{-1} K_{21}\right) \widehat{Y} \in S$, so $\widehat{K}_{22}=I-\left(I+L \Delta^{-1} K_{21}\right) \widehat{M}^{-1} \in S$.

$I-\widehat{K}_{22}-L \widehat{K}_{12}=I-I+\left(I+L \Delta^{-1} K_{21}\right) \widehat{M}^{-1}-L \Delta^{-1} K_{21} \widehat{M}^{-1}=$ $\widehat{M}^{-1}$ is invertible in $S, \widehat{\Delta}^{-1} L=\widehat{M} \cdot \widehat{M}^{-1} \widehat{N}=\widehat{N} \in S$. By Theorem 19(a), $\widehat{K}$ stabilizes $L$.

Notice that if a canonical $K$ stabilizes $L$ with internal loop, then $K_{21}$ and $I-K_{22}$ are left coprime, since $\left(I-K_{22} \Delta^{-1}\right)-$ $K_{21} L \Delta^{-1}=I$. Theorem 20 has a dual statement for rightcoprime factorizations $K$.

There is a similar result for the dual canonical controller.

Theorem 21. If the dual canonical controller $\left(\begin{array}{cc}0 & \widehat{K}_{21} \\ I & \widehat{K}_{22}\end{array}\right)$ stabilizes $L$, then $L$ can be stabilized by the dual canonical controller $\left(\begin{array}{c}0 \\ M^{-1} \widehat{K}_{12} \widehat{\Delta}^{-1} I-M^{-1}\left(I+\widehat{K}_{12} \widehat{\Delta}^{-1} L\right)\end{array}\right)$.

The proof of Theorem 21 is similar to that of Theorem 20, and we omit it.

\section{Conclusion}

In this paper, we investigate the dynamic stabilization of a large class of transfer functions in the framework of nest algebra. To obtain a natural generalization of dynamic stabilization, we introduce a new concept of stabilization by a controller with internal loop. The concept enables a simple Youla parameterization and has some advantages which turn out to be very important for infinite-dimensional systems. It makes the theory of dynamic stabilization simpler and more natural.

We also analyze canonical and dual canonical controllers, which are controllers with internal loop of a special (simple) structure. We have found that these are closely related to (doubly) coprime factorization, and we have given a complete parameterization of all stabilizing controllers with internal loop which are (dual) canonical.

\section{Conflict of Interests}

The authors declare that there is no conflict of interests regarding the publication of this paper.

\section{References}

[1] M. De la Sen, "Stability of switched feedback time-varying dynamic system based on the properties of the gap metric for operators," Abstract and Applied Analysis, vol. 2012, Article ID 612198, 17 pages, 2012.

[2] M. De La Sen, "On the characterization of hankel and toeplitz operators describing switched linear dynamic systems with point delays," Abstract and Applied Analysis, vol. 2009, Article ID 670314, 34 pages, 2009.

[3] A. Feintuch, Robust Control Theory in Hilbert Space, Springer, New York, NY, USA, 1998.

[4] G. E. Dullerud and F. Paganini, A Course in Robust Control Theory, Springer, 2000.

[5] O. Pastravanu and M.-H. Matcovschi, "Stability of matrix polytopes with a dominant vertex and implications for system 
dynamics," Abstract and Applied Analysis, vol. 2013, Article ID 396759, 11 pages, 2013.

[6] S.-Y. Li, C.-H. Yang, L.-W. Ko et al., "Implementation on electronic circuits and RTR pragmatical adaptive synchronization: time-reversed uncertain dynamical systems' analysis and applications," Abstract and Applied Analysis, vol. 2013, Article ID 909721, 10 pages, 2013.

[7] R. F. Curtain, G. Weiss, and M. Weiss, "Stabilization of irrational transfer functions by controllers with in- ternal loop, System, approximation, singular integral operators and related topics," in International Workshop on Operator Theory and Applications, IWOTA, Advances in Operator Theory and Applications, A. A. Borichev and N. K. Nikolski, Eds., pp. 179-207, Birkhäuser, Basel, Switzerland, 2000.

[8] G. Weiss and R. F. Curtain, "Dynamic stabilization of regular linear systems," IEEE Transactions on Automatic Control, vol. 42, no. 1, pp. 4-21, 1997.

[9] L. Ma, Z. Wang, Y. Bo, and Z. Guo, "A game theory approach to mixed $\mathrm{H}_{2} / \mathrm{H}_{\infty}$ control for a class of stochastic time-varying systems with randomly occurring nonlinearities," Systems and Control Letters, vol. 60, no. 12, pp. 1009-1015, 2011.

[10] J. Hu, Z. Wang, H. Gao, and L. K. Stergioulas, "Probabilityguaranteed $H_{\infty}$ finite-horizon filtering for a class of nonlinear time-varying systems with sensor saturations," Systems and Control Letters, vol. 61, no. 4, pp. 477-484, 2012.

[11] G. Wei, Z. Wang, and B. Shen, "Error-constrained finite-horizon tracking control with incomplete measurements and bounded noises," International Journal of Robust and Nonlinear Control, vol. 22, no. 2, pp. 223-238, 2012.

[12] B. Shen, Z. Wang, H. Shu, and G. Wei, " $H_{\infty}$ filtering for uncertain time-varying systems with multiple randomly occurred nonlinearities and successive packet dropouts," International Journal of Robust and Nonlinear Control, vol. 21, no. 14, pp. 16931709, 2011.

[13] Y. Chen, W. Bi, and W. Li, "New delay-dependent absolute stability criteria for Lur'e systems with time-varying delay," International Journal of Systems Science, vol. 42, no. 7, pp. 1105-1113, 2011.

[14] M. de la Sen and A. Ibeas, "On the global asymptotic stability of switched linear time-varying systems with constant point delays," Discrete Dynamics in Nature and Society, vol. 2008, Article ID 231710, 31 pages, 2008.

[15] Y. Lu and C. Shi, "Stabilization of time-varying system by controller with internal loop," Mathematical Problems in Engineering, vol. 2012, Article ID 132597, 16 pages, 2012.

[16] J. S. Baras, "Frequency domain design of linear distributed system," in Proceedings of the 19th IEEE Decision and Control Conference, pp. 728-732, December 1980.

[17] R. Curtain, G. Weiss, and M. Weiss, "Coprime factorization for regular linear systems,” Automatica, vol. 32, no. 11, pp. 1519-1531, 1996.

[18] S. Townley, G. Weiss, and Y. Yamamoto, "Diseretizing continuous-time controllers for infinite-dimensional linear systems," in Proceedings of the MTNS Symposium, pp. 547-550, Padova, Italy, July 1998. 


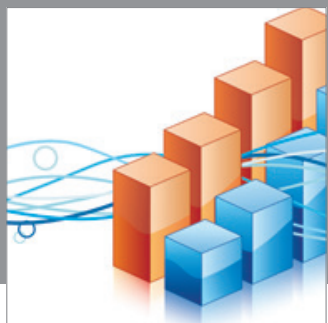

Advances in

Operations Research

mansans

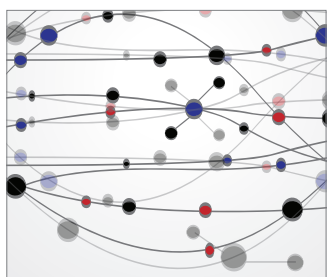

The Scientific World Journal
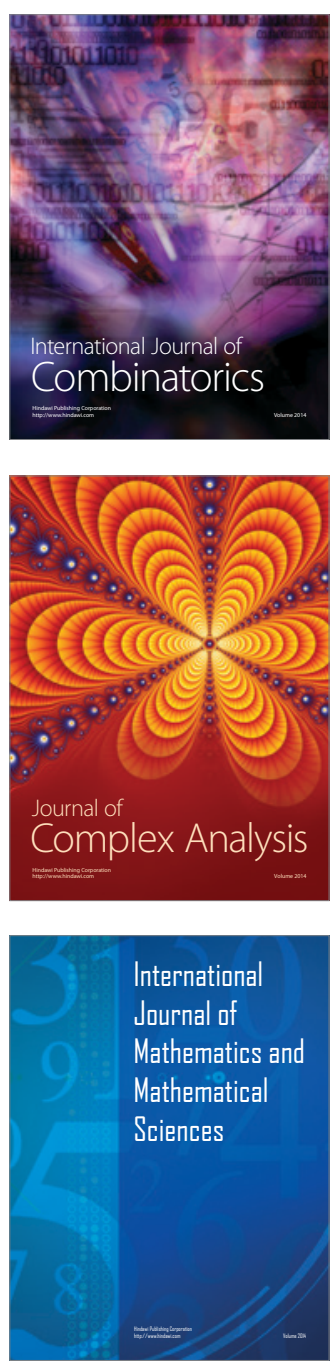
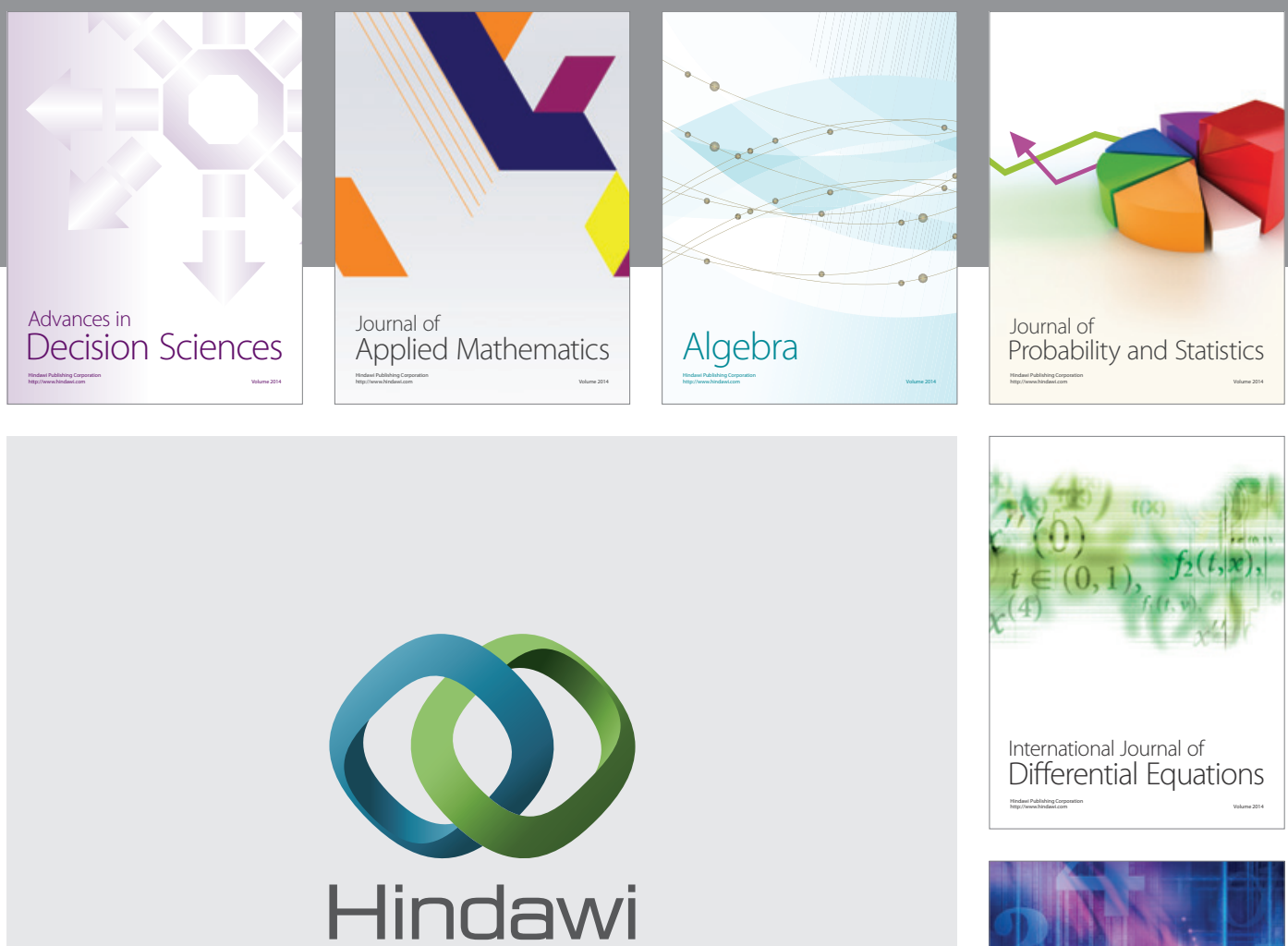

Submit your manuscripts at http://www.hindawi.com
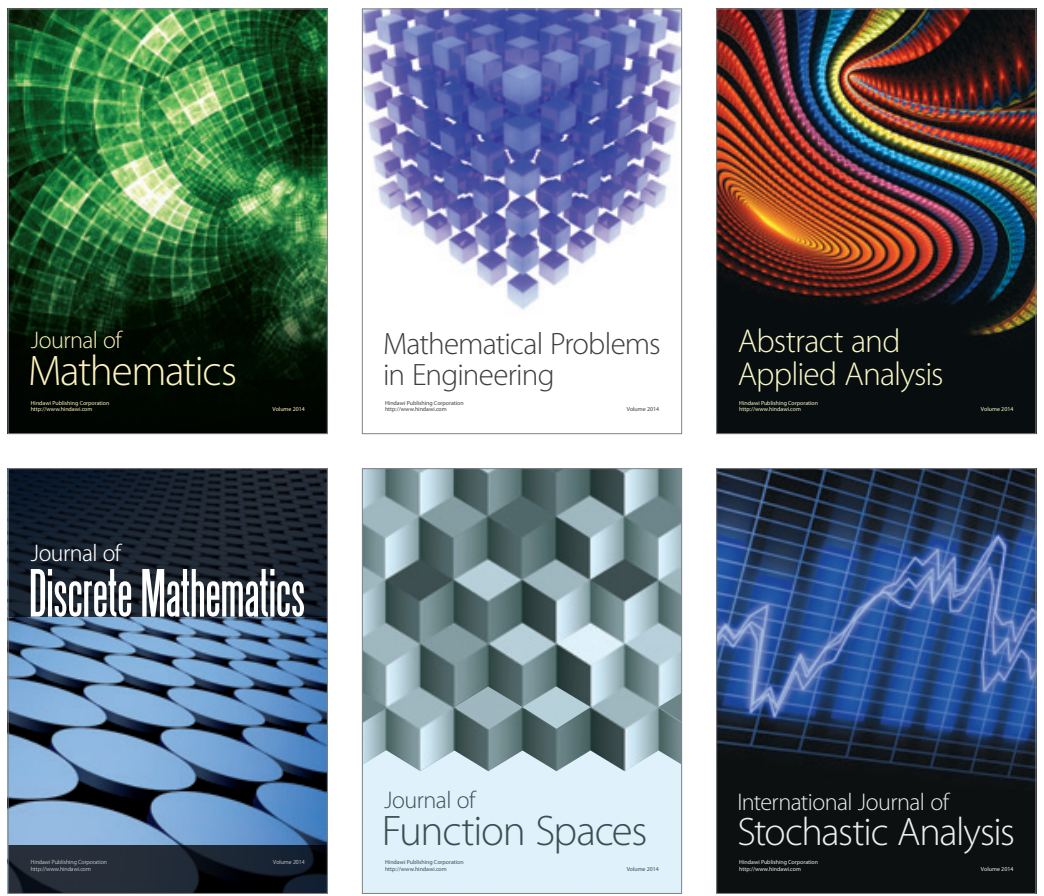

Journal of

Function Spaces

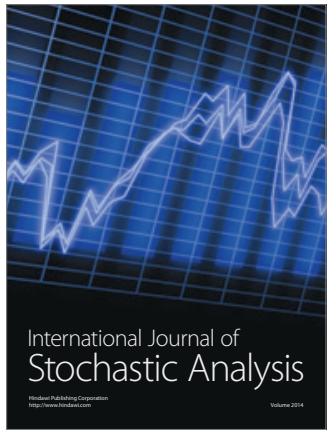

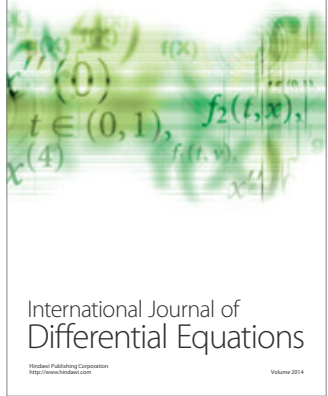
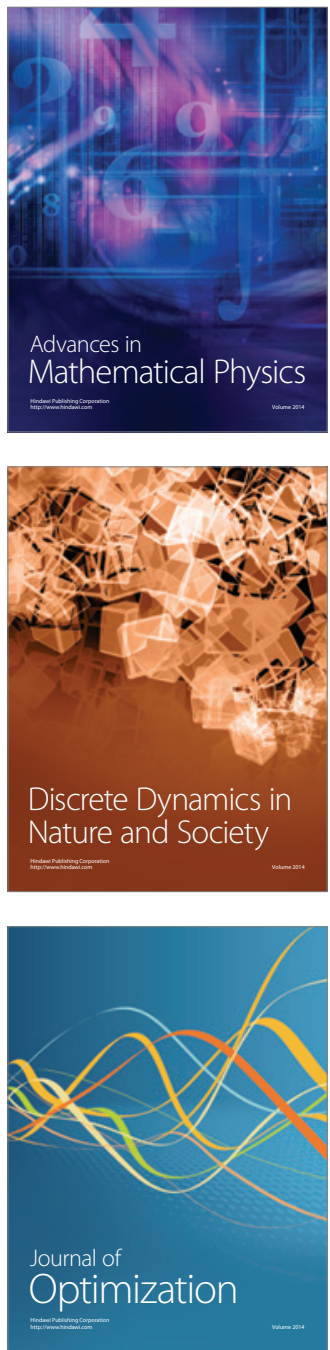